\title{
Remembering rhythm and rhyme: memorability of narratives for science communication
}

\section{Aquiles Negrete}

Centro de Investigaciones Interdisciplinarias en Ciencias y Humanidades, CEIICH-UNAM, Mexico City, Mexico

Correspondence: Aquiles Negrete (aqny @unam.mx)

Received: 29 March 2020 - Discussion started: 15 June 2020

Revised: 12 November 2020 - Accepted: 19 November 2020 - Published: 18 January 2021

Abstract. Once upon a time, narratives were considered to be a non-reliable way of representing and communicating science. Nowadays, narratives are widely accepted as an accurate way of conveying science; they represent an effective emotional trigger, a lasting memory structure and a powerful aid for learning. To study how memorable different ways of presenting information can be is a fundamental task for science communication in order to evaluate materials that not only need to be understood by the general public but also need to be retained in the long term as a part of the communication process. In this paper, I will give a brief introduction to cognitive psychology and the study of memory in relation to narratives.

Evidence from the field of memory studies suggests that narratives represent a good recall device. They can generate emotion, and this in turn is a way of focusing attention, promoting rehearsal in memory and inducing long-term potentiation. Similarly, a story produces semantic links that might assist in storing and retrieving information from memory. Studies suggest that memory span and paired-associate recall have implications in storing and recalling narratives. Evidence also suggests that the use of stories as modelling tools can organise information, provide schemas and allow extrapolation or prediction. Finally, literature in memory suggests that narratives have value as mnemonic devices.

Every man's memory is his private literature.

Aldous Huxley

\section{Introduction}

The question of how knowledge can be presented to the public in order to convey as much information as possible with maximum fidelity is a central one for science communication (Dornan, 1990; Durant et al., 1989). Memory is one possible way of assessing learning (Sternberg, 2003) and, therefore, of judging the successful communication of information. Studying how memorable different text formats are represents a fundamental task for science communication in order to produce materials that are not only expected to be understood by individuals but also stored in the long-term memory.

Much of the information that we store in our memory is not acquired first hand, through personal experience, but second hand, through reading or listening to other people talk about their experiences (Cohen, 1989). Memory for spoken information and memory for written information differ in important ways. Reading is a private and solitary occupation; it has no conversational context such as intention, intonation, gesture, facial expression or personality of the speaker. Written material has to be more formally structured and must conform to certain rules and formats to be intelligible to a wide range of potential readers.

In general, we remember meaning better than wording (Cohen, 1989). The general rule for narratives (short stories, drama, comics, novels, etc.) appears to be that the meaning, the gist, the most important and most relevant facts are preserved by the memory (Cohen, 1989). Almost any material becomes easier to remember if it is included in a narrative (Bruner, 1986, 1990; Crowley, 2018; ElShafie, 2018). There are several factors concerning memory that make narrative a lasting structure; some of them are related to the memory 
process itself and others to the intrinsic characteristics of narratives as a means of expressing information.

\section{Objective and methodology}

The objective of this work is to provide a literary review of memory studies regarding memorability of narratives.

In previous work (Negrete, 2009, 2013, 2014; Negrete and Lartigue, 2010; Rios and Negrete 2013; Lartigue and Negrete 2016), we provided empirical evidence suggesting that science can be communicated and learnt through narratives, and that this represents a more enjoyable way of learning compared to traditional texts. In particular, we found that narrative information is retained for lengthier periods than factual information in long-term memory. Our evidence suggested that narratives constitute an important means for science communication to convey information in an accurate, memorable and enjoyable way.

In this opportunity, my aim is to examine what has been reported in literature regarding features of the memory process that contribute to making narratives a memorable device. Although narratives have implications in short-term memory processes, I will concentrate on long-term memory, the most relevant features for science communication.

\section{Narrative representation}

A dominant model of rationality implies a single type of discourse, one that puts forward hypotheses, reported evidence and systematically inferred conclusions. Stories, in contrast, frequently carry the connotation of falsehood or misrepresentation (Bruner, 1986). However, several authors acknowledge that many scientific and mathematical hypotheses emerge as little stories or metaphors. In Howard's (1991) view, there is a relationship between science and storytelling. This author considers, for instance, that science represents an example of constructing meaning through storytelling.

Bruner (1986) originally proposed two modes of cognitive functioning, namely paradigmatic and narrative. Each provides a different way of organising experience, constructing reality and communicating knowledge. They are, at the same time, complementary and irreducible to one another. While paradigmatic knowledge is focused on what is common among items, narrative knowledge focuses on the particular and special characteristics of actions. Human action is the result of the interrelation of previous learning, experience and present and future expectation. While paradigmatic knowledge is carried in individual words that name a concept, narrative knowledge is maintained in stories with plot. Storied memories retain the complexity of the situation in which an action was undertaken and the emotional and motivational meaning connected with it. The collection of storied experiences provides a basis for understanding new ac- tion episodes by means of analogy (Amos and Wisniewski, 1995).

Narratives can take different forms. Among the different types of narratives, parables and myths have a particular interest for science communication. Both are aids in understanding difficult concepts. Although the latter may not match our current sense of reality, they can be used in science communication to analyse the values and limits of scientific knowledge (Blades, 2001). Also, science fiction is of paramount importance in science communication as it is the literary genre most frequently used to represent, explore and play with science. Science can be used as the subject of the narrative, as the basis for the plot, as a background or setting or even as a metaphor (Willis, 1998). Science fiction represents a valuable tool for science education (Gough, 1993; Appelbaum, 1995) and communication.

For this work, a narrative is a particular type of discourse production in which events and actions are assembled in an organised unity with the help of an intrigue (Connelly and Clandinin, 1990). Narrative texts answer the question of "What happened?" Characters, events and plot exist in a world where time goes by (Amos and Wisniewski, 1995). According to the cognitive model, narratives can be seen as memory-enhancing devices (Atkinson and Shiffrin, 1971).

\section{Literary review on memory studies}

\subsection{Cognitive psychology}

Cognition is a sub-discipline of psychology that studies how humans perceive, learn, remember and think about information (Sternberg, 2003). Memory is the means by which humans retain and draw upon past experience and use this information in the present (Tulving and Craik, 2000). It is the record of experience that underlies learning. Learning can be defined as a biological mechanism that permits us to face a changing world, i.e., it is a process by which long-lasting changes in the behaviour potential take place as a result of experience.

In cognitive psychology, the following three main memory operations are distinguished: (i) encoding, (ii) storage and (iii) retrieval (Baddeley, 2000). Each operation represents a stage in memory processing. Through encoding, the individual transforms sensory data into a form of mental representation; through storage, the encoded information is maintained in the memory, and through retrieval, it is pulled out for use. Pioneering work by Tulving and Pearlstone (1966), and Murdock (1961), suggested that although encoding, storage and retrieval phenomena are theoretically clearly defined, in practice there is considerable overlap, and they are therefore too interdependent to allow for working with each as a separate unit. 


\subsection{Long-term memory}

There are different ways of encoding in long-term memory (LTM; Sternberg, 2003; Crowley, 2018). Most information stored in long-term memory seems to be semantically encoded. There is evidence in early work on the area that other forms of encoding exist in long-term memory, such as visual encoding (Frost, 1972) and acoustic encoding (Nelson and Rothbart, 1972), but they play a minor role in comparison to semantic encoding.

Information from short-term memory is transferred to long-term memory, depending on whether the information involves declarative (declarative knowledge refers to recalling facts) or non-declarative memory. Some forms of non-declarative memory, like priming and habituation, are ephemeral and dissipate rapidly; others, such as procedural and conditioning, are maintained for longer periods, especially when rehearsed. For declarative knowledge to enter into LTM, two main processes are involved, namely attention and association (of new information with previous knowledge and also of schemas). The process of integrating new information into stored information is referred to as consolidation (Squire, 1986).

Retention and enhancement of memory during consolidation can be promoted with different meta-memory strategies (Koriat and Goldsmith, 1996; Metcalfe, 2000). These strategies involve a conscious act of reflection by rehearsing and organising (mnemonics) new information destined to stay in long-term memory.

\subsubsection{Long-term potentiation and rehearsal}

Every experience leaves a trace in the brain. Every experience is potentially a memory, but only some traces seem to become permanently imprinted into brain tissue. Every experience - whether it is a real or perceived event, a thought, a feeling, a fragment of the imagination or a recollection of a previous experience - involves the activation of a unique neural firing pattern (Maren, 1999). Some events produce strong and long-lasting patterns which tend to recur continually. When connections are repeatedly activated, they form even more robust links which bind them into a single unit called long-term potentiation (LTP). Research suggests that memories generated in this way (LTP) can last a lifetime (Bahrick and Hall, 1991).

Rehearsal is perhaps the simplest and most effective strategy that can be used in a memory task. It is an interactive process by which information in short-term memory is continually articulated or refreshed. Its importance is that it maintains information in short-term memory by ensuring a sufficiently high level of activation, and it facilitates the transfer of information to long-term memory and subsequent retrieval by allowing additional time for more elaborate item processing (Dempster, 1981).
There are three important moments for long-term potentiation (long lasting memory), namely attention, emotional response and rehearsal. It is interesting to note that a typical joke relayed orally (normally the narrative of something funny happening to somebody) concentrates these three elements. When someone is going to tell a joke, people pay attention to the speaker. If the joke is good, they laugh (emotional response). Hours later, or even the next day, when people remember the joke, they will probably laugh again (rehearsal). That is the way people learn jokes and reproduce them with friends and colleagues. The joke has a precise structure in order to be funny. It is interesting how we are able to remember such a structure with remarkable fidelity so that we are able to retell the joke with the precision required to make people laugh. Humorous narratives should be considered as an important resource for science communication as they represent a tool that can induce long-term potentiation by promoting attention, emotional response, and rehearsal (see, for example, Primo Levi's (1985) narrative in Sect. 4.6).

\subsubsection{Oblivion}

Oblivion is defined as the decline in performance after learning. It occurs after a certain period. To measure it, researchers observe behaviour after a period in which the learnt behaviour has not taken place (retention period).

It is worth noting that oblivion occurs quickly when we learn lists of unrelated words or unsystematic items. In contrast, if the text is meaningful, it is more likely that we will remember it for longer periods. Previous knowledge (proactive knowledge) can also reduce oblivion (Squire, 1986). Pioneering work by Sir Frederick Bartlett (1932) showed that a story which was difficult to understand was made modern and comprehensible by participants thanks to proactive knowledge. His experiments consisted of presenting an indigenous North American story called The War of Ghosts to a group of participants in Britain. Bartlett found that his participants distorted their recall to provide a story that was more comprehensive to them. Their previous knowledge and expectations had a substantial effect on their recollection. In so doing, Bartlett developed the idea that in memory tasks we use our already existing schemas, which affect the way we recall and learn. In the geosciences context, it has been suggested that myths (a form of narratives) help in reducing the oblivion of geological hazards (flooding, eruptions and earthquakes), and this proactive knowledge has helped to create a culture of prevention in different human groups (Crowley, 2018; Lanza and Negrete, 2007). One interesting example is the myths concerning exploding lakes, such as Lake Nyos in Cameroon. As Shanklin (2007) reports, many stories were based on the assumption that lakes are the homes of ancestors and spirits and can be source of death. 


\subsection{Emotion and attention}

Experiencing emotion provides a basis for simple learning and memory (Sternberg, 2003). Emotional learning and memory, such as fear conditioning, are simple forms of associative learning that support the acquisition of knowledge; it is acquired rapidly and retained over long periods (Maren, 1999). An effect of emotional stimulation is to direct attention towards the events that provoked it. This attention in turn augments the brain activation associated with the event. Attention is effectively the first stage of laying down memory (Rupp, 1998).

Evidence shows that what distinguishes enduring experiences from those that are lost is that when they occurred they either created or coincided with higher-than-normal levels of emotion (Baddeley, 1997). It is clearly vital for humans to remember events that are emotionally charged because they are likely to be important ones. They can be used to guide present and future actions. They can be used, for example, to avoid danger (such as geological hazards) or to steer us towards a desirable outcome (O'Brien, 2000). Interestingly, the same neurochemicals that are released into the bloodstream to put the body on alert also instruct the brain to store a lasting record of the moment. This is the case for acetylcholine, noradrenaline, dopamine and glutamate, which all participate in the creation of links between neurons (Rupp, 1998; Zak, 2007).

Durability of a particular memory seems to depend on how exciting the original experience was (or how excited the individual's brain was when it occurred), how much attention was paid to it and how often it is recalled (Sternberg, 2003). In Lotman (1990)'s words, "narratives are a way of expressing ideas and amplifying emotions". If emotions are generated, there is a greater opportunity to concentrate attention, and the possibility to produce long-term potentiation is higher. Also, the possibility to rehearse the emotions is greater, since we tend to repeatedly remember passages that result from a meaningful or emotional experience (Sternberg, 2003).

\subsection{Memory in context and knowledge networks}

According to Gough (1993), context is of paramount importance in order to understand memory process. No subject exists in isolation. Knowledge does not remain neatly compartmentalised into disciplines but spills over and transgresses boundaries. Everything that happens has a context, not only circumstances and surroundings but also internal states, emotions and physical feelings. If an event is laid down as a memory, some of its context is laid down with it and becomes a hook for remembering (Rupp, 1998). Contextual elements can be valuable aids to recall because when one part of a memory is retrieved, it often hooks out all the others.

Memories that have similar connotations, forming links based on meaning, are called semantic links. Semantic links act like a cross-referencing system; once we have found a useful piece of information, we can connect it with many more that might also be relevant (Cohen, 1989). Memories that are formed simultaneously are linked by association. These associative links are fundamental to our understanding of the world and often allow us to make predictions based on previous experience (see also Sects. 4.9 and 4.10). Most of the time, semantic and associative links work unconsciously; as soon as one concept is activated in memory, activation spreads automatically to other ideas related through meaning or past experiences.

A story can be seen as an expressive device that, by means of a plot, associates characters, situations, places and information to produce semantic links and a cross-referencing system that can assist in storing and retrieving information in, and from, memory (i.e. scientific knowledge).

\subsection{Human memory systems}

According to Tulving (1972), there are six major human memory systems, namely semantic, episodic, procedural, perceptual, representational and short-term memory. There is reasonable evidence for the existence of the first two types, namely semantic and episodic memory.

With the aid of semantic memory, individuals are able to register and store information about the world in the broadest sense (i.e. not personally experienced) and are capable of retrieving it. Semantic memory allows people to think about things that are absent to the senses at the time (Tulving, 1972). Semantic memory is automatic, i.e. it does not require a conscious recollection. It develops earlier in childhood than episodic memory (Tulving, 1972).

\subsection{Episodic memory}

This is the type of memory used to remember events in our lives. Therefore, episodic memory is related to personal experiences in subjective space and time. An episodic memory consists of memories that come from different areas of the brain that are bound together to create an episode rather than a collection of impressions or items of knowledge (Crowley, 2018). In contrast to semantic memory and other kinds of memory systems, in this case the individual is able to be transported into the personal past and future at will (Tulving, 2000). In times of crisis, the individual is able to bring the past to the forefront in order to reinterpret the events of a lifetime.

Tulving (1966) pointed out that retrieving information from each memory system is associated with distinct memory awareness experiences. According to this author, when an individual uses episodic memory, they are conscious of remembering past experiences, whereas, in the case of semantic memory, a person's conceptual knowledge is characterised by memory awareness involving feelings of familiarity or just knowing. 
Episodic memory is characterised by the following two aspects of temporal structuring: the location of the event in a specific past time in relation to the present and a temporal sequencing within the episode remembered (Nelson and Rothbart, 1972). Both of these aspects rely on a sense of the extended self, and apparently, the role of autobiographic memory is to provide a sense of continuity of the self across time from past to future (Nelson and Rothbart, 1972).

There is a strong link between episodic memory and emotions. The way in which memories are formatted determines their emotional significance and the retrieval pathways to other episodic memories. Earlier experiences tend to be recalled from a third person point of view (i.e. as an observer), while more recent events are usually recalled from the first person point of view (i.e. as a participant). Emotions are usually stronger when memories are recalled from a participant's point of view, while the observer's point of view tends to be more objective.

Narratives offer information that is contextualised in reallife situations (episodes). When an episode in a narrative work evokes emotion in the reader, this incident may become memorable. Narratives (fictional or non-fictional) provide the opportunity to create episodes. If the narrative episode evokes emotions and part of it contains science, then it would be reasonable to expect that information contained in it (included science) will form a lasting memory.

The following narrative is a shortened version (performed by the author) of Primo Levi's Nitrogen (1985). It provides an example of an episode that includes science and has proved to be a memorable device (Negrete, 2009).

The client explained to me that he was the owner of a cosmetics factory, and he wanted to produce a certain kind of lipstick. He needed a few kilos of alloxan. He would pay a good price for it, provided I committed myself by contract to supply it only to him. He had read that alloxan in contact with the mucous membrane confers on it an extremely permanent red colour because it is not a superimposition, in short, a layer of varnish like lipstick, but a true and proper dye, as used on wool and cotton. I gulped, and, to stay on the safe side, replied that we would have to see; alloxan is not a common compound nor very well known - I don't think my old chemistry textbook devoted more than five lines to it - and at that moment I remembered only vaguely that it was a derivative of urea and had some connection with uric acid. I dashed to the library at the first opportunity and hastened to refresh my memory as to the composition and structure of alloxan.

Alloxan is a hexagonal ring of oxygen, carbon, hydrogen and nitrogen; it is a pretty structure! It makes you think of something solid, stable, well linked. In fact, it happens also in chemistry as in architecture that "beautiful" edifices, that is, symmetrical and simple, are also the most sturdy: in short, the same thing happens with molecules as with the cupolas of cathedrals or the arches of bridges. Alloxan was known for almost 70 years, but as a laboratory curiosity; the preparation method described had a pure academic value and was made from expensive raw materials, which (in those years right after the war) it was optimistic to hope to find on the market. The sole accessible preparation was the oldest: it did not seem too difficult to execute and consisted in an oxidising demolition of uric acid. Just that - uric acid, the stuff connected with gout, intemperate eaters and stones in the bladder. It was a decidedly unusual raw material but perhaps not as prohibitively expensive as the others.

Subsequent research taught me that uric acid, very scarce in the excreta of man and mammals, constitutes, however, 50 percent of the excrement of birds and 90 percent of the excrement of reptiles. Fine. I phoned the client and told him that it could be done; he just had to give me a few days' time. Before the month was out I would bring him the first sample of alloxan and give him an idea of the cost and how much of it I could produce each month. The fact that alloxan, destined to embellish ladies' lips, would come from the excrement of chickens or pythons was a thought which didn't trouble me for a moment. The trade of chemist teaches you that matter is matter, neither noble nor vile, infinitely transformable, and its proximate origin is of no importance whatsoever. Nitrogen is nitrogen, it passes miraculously from the air into plants, from these into animals, and from animals to us; when its function in our body is exhausted, we eliminate it, but it still remains nitrogen, aseptic, innocent. We - I mean to say we mammals who in general do not have problems about obtaining water, have learnt to wedge it into the urea molecule, which is soluble in water, and as urea we free ourselves of it; other animals, for whom water is precious (or it was for their distant progenitors), have made the ingenious invention of packaging their nitrogen in the form of uric acid, which is insoluble in water, and of eliminating it as a solid, with no necessity of having recourse to water as a vehicle.

I returned home that evening and informed my wife that the next day I would leave on a business trip: that is, I would get on my bike and make a tour of the farms on the outskirts of town in search of chicken shit. She did not hesitate; she would come along with me. But she warned me not to have too many illusions. Finding chicken shit in its pure 
state would not be so easy. In fact, it proved quite difficult. First of all, the pollina - that's what the country people call it, which we didn't know, nor did we know that, because of its nitrogen content, it is highly valued as a fertiliser for truck gardens - the chicken shit is not given away free; indeed, it is sold at a high price. Second, whoever buys it has to go and gather it, crawling on all fours into the chicken coops and gleaning all around the threshing floor. And third, what you actually collect can be used directly as a fertiliser but lends itself badly to other uses. It is a mixture of dung, earth, stones, chicken feed, feathers and chicken lice, which nest under the chickens' wings. In any event, paying not a little, labouring and dirtying ourselves a lot, my undaunted wife and I returned that evening with a kilo of sweated-over chicken shit.

The next day I examined the material: there was a lot of gangue, yet something perhaps could be obtained from it. But simultaneously I had an idea; just at that time, in the Turin subway gallery, an exhibition of snakes had opened. Why not go and see it? Snakes are a clean species, they have neither feathers nor lice, and they don't scrabble in the dirt. And besides, a python is quite a bit larger than a chicken. Perhaps their excrement, at 90 percent uric acid, could be obtained in abundance, in sizes not too minute and in conditions of reasonable purity. This time I went alone: my wife is a daughter of Eve and doesn't like snakes. The director and the various workers attached to the exhibition received me with stupefied scorn. Where were my credentials? Where did I come from? Who did I think I was showing up just like that, as if it were the most natural thing, asking for python shit? Out of the question, not even a gram; pythons are frugal, they eat twice a month and vice versa; especially when they don't get much exercise. Their very scanty shit is worth its weight in gold; besides, they and all exhibitors and owners of snakes - have permanent and exclusive contracts with big pharmaceutical companies. So get out and stop wasting our time. I devoted a day to a coarse sifting of the chicken shit, and another two trying to oxidise the acid contained in it into alloxan. The virtue and patience of ancient chemists must have been superhuman, or perhaps my inexperience with organic preparations was boundless. All I found were foul vapours, boredom, humiliation and a black and murky liquid which irremediably plugged up the filters and displayed no tendency to crystallise as the text declared it should. Best to return among the colourless but safe schemes of inorganic chemistry.

\subsection{Mnemonics}

Before the invention of writing, and long afterwards in many cultures, stories were sung or recited from memory. Rhythm, rhyme and melody were used to provide a framework that aided in their memorisation. Mnemonics are one tool employed to aid recitation from memory. It is defined as the art of improving memory, or a system to aid the memory, i.e. any strategy that helps people remember. It normally means signals for learning that will later induce a memory of the experience (Sternberg, 2003).

According to Lotman (1990), mnemonics can be seen as a way of internal communication that is made up of messages to the self, with the purpose of retaining information, and includes different sorts of memoranda and reminders. Essentially, such reminder devices add meaning (or personal meaning) to otherwise meaningless, unrelated or arbitrary lists of items for the individual. Mnemonics superimpose an artificial, logical structure (which can be seen as a model) on data, which are not necessarily related. A mnemonic device can be an image (e.g. "Alloxan is a hexagonal ring of oxygen, carbon, hydrogen and nitrogen; it is a pretty structure! It makes you think of something solid, stable, well linked. In fact, it happens also in chemistry as in architecture that 'beautiful' edifices, that is, symmetrical and simple, are also the most sturdy: in short, the same thing happens with molecules as with the cupolas of cathedrals or the arches of bridges"; Levi, 1985), an acronym, a verse, a rhyme (e.g. "matter is matter, neither noble nor vile"; Levi, 1985), a peg word, a catchphrase or a story that helps us to remember (Luria, 1986).

In Yates' (1992) view, a feature of Cosmae Rosselli's book (Thesaurus artificiosae memoriae) is the mnemonic verses given to help memorise the orders of places in hell or the order of the signs of the zodiac. These verses were written by a Dominican inquisitor. These songs or chants by the inquisitor constitute an interesting example of the use of artificial memory via mnemonics (Yates, 1992).

Most of the world's great religions have strong oral traditions in which sacred texts are memorised in their entirety for prayer and to preserve them for posterity. For example, in the Mishnah, the Jewish written record of the oral law, some literary resources such as metaphors, digressions and poetic images can actually be viewed as mnemonic aids. The Qur'an also contains mnemonic aids. This religious book was written both as a work of rhythmic prose and as an epic poem; thus, rhythm, rhyme and meaning connect every word, making it memorable (Luria, 1986).

Narratives can be seen as mnemonic structures that superimpose an artificial, logical structure on data which is not necessarily related. In this way, scientific factual information can be communicated by being embedded in a mnemonic structure (the story), which facilitates future recollection. 


\subsection{Memory span and paired-recall association}

In early work in this area, Dempster (1981) defined memory span as the maximum length of a series of words, images or items that can be reproduced at different stages in time. One of the most practical and important implications of memory study is in education. As short-term memory span is indicative of overall intellectual ability, it can be used as a diagnostic tool both for helping educators (and communicators) to adapt teaching (and learning materials) to the specific needs of the learner and for measuring improvements in intellectual ability Dempster (1981). Higher spans are the result of grouping and organisation (Estes, 1974). Organisation, in turn, is one of the key elements of paired-recall association.

Pioneering work by Epstein, Rock and Zuckerman (1960) suggested that when two objects have been perceived or imagined to be interacting, recalling the name of one in response to the name of the other is more frequent than when the objects have been perceived or imagined to be side by side. This effect in memory is called paired-recall association. As a result of the relationship between two objects, they develop certain properties and interactions. A relation or interaction constitutes a feature that characterises both objects, which enables the individual to retrieve one when the other is provided (Wilton, 1989). An interesting example of the effectiveness of rhythm and paired-recall association as mnemonic aids is clear when we try to remember the lyrics of a song, and it suffices to recollect its rhythm in order to do so.

When words are used as units of meaning, the semantic components of the words are activated (Wilton, 1990). If two words are associated semantically, this ensures that common structures are activated in that task. Therefore, in the search for recall, the items to be recalled are found together. On the other hand, when words are used as a collection of symbols without semantic meaning, the common structures are not activated, and recall is disorganised.

Following this line of argument, it would be plausible that stories represent a means of increasing memory span, a way to facilitate retrieval from memory by paired recall association and a powerful device to convey science to the general public in a long-lasting way.

\subsection{Models}

According to the classical work by Giere (1979), models can be classified into the following three categories: scale models that represent reality to a particular scale, analogue models, which are useful for understanding other proposed new models, and theoretical models, the most abstract form of a model as they are imaginary and often explained with analogical models. Examples of the latter are the thought experiments. A thought experiment is an idealisation or abstraction of existing physical conditions. A thought experiment implies the use of visual imagery abstracted from phenom- ena that we have actually experienced. This imagery allows intuition, an impression of how things are connected, innovation and the possibility of modelling in the mind. This kind of thinking was used by famous scientists such as Galileo, Einstein, Maxwell, Bohr and Heisenberg.

In Casti's (1993) view, models can serve three purposes; they can be predictive, explanatory and prescriptive. Prescriptive models give us the opportunity not just to explain or predict but also to manipulate some aspect of the world through handles on the model (Casti, 1993).

Casti (1993) compares modelling with painting and other artistic disciplines. When an artists paint, they never create on canvas the exact image of the subject in front of them. Instead, they try to capture the essence of meaningful characteristics so that the viewer is able to know more about the object painted than from looking at the real thing. In this sense, the object of the art (painting, sculpture, music or literature) shows hidden characteristics by using magnifying glasses, special lights, tones, rhythms or narrative resources. Giere's (1979) and Casti's (1993) arguments claim that stories can be seen as narrative models that have the power to explain, the capacity to show scale, an ability to predict the future, to produce analogies and metaphors as well as to theorise.

Yuri Lotman (1977) suggested that semiotic systems are models that explain the world in which we live. Amongst all semiotic systems, language is the primary modelling system in which we comprehend the world by means of the model that it provides. Myth, cultural rules, religion, paint, music, literature (narratives) and science are secondary modelling systems. All of them are of equal interest as models for understanding and talking about the world.

In Johnson-Laird (1983)'s words, “... stories are represented as mental models in the reader's mind". To construct a mental model of a story is to imagine what was happening in the narrative. A mental model is a global representation that integrates information from different parts of the story. It is constructed as the story unfolds and represents the scene, characters and events, incorporating spatial, temporal and casual relations (Johnson-Laird, 1983). Mental models have the intuitively appealing feature of treating memory for stories and memory for real-world events as essentially the same (Yates, 1992).

Narratives can also be seen as secondary modelling systems in which information is represented and organised by means of a plot. This enables us to make sense of reality and prepare information in an organised structure ready for future recall. Stories can be seen as narrative models as they depict the model which has the capacity to explain. For example, in the capacity to show scale, as in Carbon by Primo Levi (1985), the possibility to show processes that take millions of years in few pages, as in The crabs take over the island by Anatoly Dneprov (1969), an ability to predict the future, as in The time machine by H .G. Wells (1895), or to produce analogies and metaphors, as in Flatland by Edwin A. Ab- 
bot (1884; Abbot, 1992) and to theorise, as in Italo Calvino's Cosmicomics (1969). Using narratives provides a powerful tool to communicate science.

\subsection{Story schemas}

One of the earliest studies of memory and narratives was carried out by Bartlett (1932). Unlike many psychologists of his day, Bartlett recognised the need to study memory retrieval with connected texts rather than studying unconnected strings of digits, words or nonsense syllables. He introduced the idea that schemas, or mental frameworks, built up from prior knowledge and experience are influential in determining and shaping the memory of a story (see Sect. 3.2.2)

During the decade of the 1970s, Bransford and Johnson (1973) challenged the idea that schemas work at a retrieval stage. They constructed texts that described a situation in such a way that the reader was unable to understand its meaning unless some clues were provided. The researchers suggested that when new information cannot be related to an appropriate schema, very little is remembered. Other researchers found similar results in comparative experiments of prose retention (see Dooling and Lachman, 1971).

Today two kinds of schemas are distinguished, namely event schemas and story schemas. Event schemas consist of knowledge about the subject matter of the story (Cohen, 1989; Christy et al, 2017). For example, the event schemas activated in remembering The man who mistook his wife for a hat by Oliver Sacks (a collection of different narratives about Oliver Sacks' patients) might include knowledge of psychiatric hospitals, admirable characters, self-identity, physiology of the brain, sensory ghosts, disembodiment or autism. Story schemas consist of abstract, content-free knowledge about the structure of a typical story.

For science communication, one of the advantages of story schemas is that the majority of people have been exposed to them since childhood in such forms as religious instruction, drama or reading fiction. Therefore, it represents a widespread and well-established knowledge held by the general public that can be used, without previous instruction, to the benefit of popularisation of science.

\section{Final note}

It is still necessary to invest a considerable amount of effort in investigating the use of narratives in science communication as it is a rather recent field - and a promising one. For instance, it is necessary to explore in more depth the adequate characteristics of narrative text for effective science communication (i.e. the use of powerful mnemonic devices). From my perspective, science communication via narratives should follow a series of rules, as it happens with other narrative subgenera, such as thrillers, horror stories, historic novels, etc. I have named these kind of narratives SciComm narratives (Negrete, 2014), and they could be considered as a new narrative subgenera with their own characteristics and rules. Therefore, it is important to generate more knowledge that enables us to provide a solid theoretical body around narratives for science communication (SciComm narratives).

Data availability. No data sets were used in this article.

Competing interests. The author declares that there is no conflict of interest.

Special issue statement. This article is part of the special issue "Five years of Earth sciences and art at the EGU (2015-2019)". It is a result of the EGU General Assembly 2016, Vienna, Austria, 17-22 April 2016.

Acknowledgements. I would like to thank Tiziana Lanza for all her generous help in making this paper possible.

Review statement. This paper was edited by Tiziana Lanza and reviewed by Nancy Longnecker and Sara J. ElShafie.

\section{References}

Abbot, E. A.: Flatland: A romance of many dimensions, Thrift Editions, New York, USA, 1992.

Amos, H. and Wisniewski, R.: Life history and narrative, The Falmer Press, London, UK, 1995.

Atkinson, R. C. and Shiffrin, R. M.: The control of short-term memory, Sci. Am., 225, 82-90, 1971.

Baddeley, A. D.: Human memory: theory and practice, Alyn \& Bacon, Minneapolis, USA, 1997.

Baddeley, A. D.: The episodic buffer: A new component of working memory, Sci.-Fiction Stud., 4, 417-423, 2000.

Bahrick, H. P. and Hall, L. K.: Lifetime maintenance of high school mathematics contents, J. Exp. Psychol., 120, 20-33, 1991.

Bartlett, F. C.: Remembering: a study in experimental and social psychology, Cambridge University Press, New York, USA, 1932.

Blades, D. W.: The simulacra of science education, in: Postmodern science educations, edited by: Weaver, J. A. and Morris, M., Peter Lang Publishing, Inc., New York, USA, 156-171, 2001.

Bransford, J. D. and Johnson, M. K.: Considerations of some problems of comprehension, in: Visual information processing, edited by: Chase, W. G., Cambridge Academic Press, UK, 1973.

Bruner, J. S.: Actual minds, possible worlds, Harvard University Press, Boston, USA, 1986.

Bruner, J. S.: Acts of meaning, Harvard University Press, Boston, USA, 1990.

Calvino, I.: Cosmicomics, Picador, London, UK, 1969.

Casti, J.: Be worlds would, John Willey \& Sons Inc., New York, USA, 1993.

Christy, K. R., Jansen, J. D., Jensen, D., Sarapin, S. H., Yale, R. N., Weaver, J., and Pokharel, M.: Theorizing the Impact of Tar- 
geted Narratives: Model Admiration and Narrative Memorability, J. Health Commun., 22, 433-441, 2017.

Cohen, G.: Memory in the real world, Lawrence Erlbaum Associates Ltd, London, UK, 1989.

Connelly, F. M. and Clandinin, J. D.: Stories of Experience and Narrative Inquiry, Educ. Res., 9, 2-14, 1990.

Crowley, K. A.: Memorability in Narration: An Overview of Mnemonic Features in Oral and Written Tradition, Research Journal: Studies about Languages, 32, 77-93, 2018.

Dempster, F. N.: Memory span: sources of individual and developmental differences, Psychol. Bull., 89, 63-100, 1981.

Dneprov, A.: The crabs take over the island, in: Russian science fiction, edited by: Magidoff, R., University of London Press, London, UK, 1969.

Dooling, D. J. and Lachman, R.: Effects of comprehension on retention of prose, J. Exp. Psychol., 88, 216-222, 1971.

Dornan, C.: Some problems of conceptualizing the issue of science and the media, Crit. Stud. Mass Comm., 7, 48-49, 1990.

Durant, J. R., Evans, G. A., and Thomas, G. P.: The public understanding of science, Nature, 340, 11-14, 1989.

ElShafie, S. J.: Making Science Meaninful for Broad Audiences through Stories, Integr. Comp. Biol., 58, 1213-1223, 2018.

Epstein, W., Rock, I., and Zuckerman, C. B.: Meaning and familiarity in associative learning, Psychol. Monogr.-Gen. A., 74, 1-22, 1960.

Estes, W. K.: Learning theory and intelligence, Am. Psychol., 29, 740-749, 1974

Frost, N.: Encoding and retrieval in visual memory tasks, Exp. Psychol., 9, 317-326, 1972.

Giere, R.: Understanding scientific reasoning, The Dryden Press, Sunders Collage Publishing, New York, USA, 1979.

Gough, N.: Laboratories in fiction: science education and popular media, Deakin University, Geelong, Australia, 1993.

Howard, G. S.: Culture tales, Am. Psychol., 19, 187-197, 1991.

Johnson-Laird, P. N.: Mental models, Harvard University Press, Boston, USA, 1983.

Koriat, A. and Goldsmith, M.: Monitoring and control processes in the strategic regulation of memory accuracy, Psychol. Rev., 103, 490-517, 1996.

Lanza, T. and Negrete, A.: From myth to earth education and science communication in Piccardi, L. and Masse, W. B., Myth and Geology, Monography published by The Royal Society of Geology of London, London, UK, 2007.

Lartigue, C. and Negrete, A.: Photocomic Narratives as a Means to Communicate Scientific Information about Use, Treatment and Conservation of Water, Modern Environmental Science and Engineering, 2, 800-808, 2016.

Levi, P.: The periodic table, Abacus, London, UK, 1985.

Lotman, M. Y.: Primary and secondary communication-modeling systems, in: Soviet Semiotics, edited by: Lucid, D. P., John Hopkins University Press, Baltimore, USA, 1977.

Lotman, M. Y.: Universe of the mind. A semiotic theory of culture, Indiana University Press, USA, 1990.

Luria, A. R.: The Mind of the mnemonists, Harvard University Press, Boston, USA, 1986.

Maren, S.: Long-term potentiation in the amygdala: a mechanisms for emotional learning and memory, Trends Neurosci., 22, 561$567,1999$.
Metcalfe, J.: Metamemory: theory and data, in: The Oxford handbook of memory, edited by: Tulving, E. and Craick, M., Oxford University Press, New York, USA, 197-211, 2000.

Murdock, B. B.: Short-term retention of single paired-associates, Psychol. Rep., 8, 280-289, 1961.

Negrete, A.: So what did you learn from the story? Science communication via narratives, VDM Verlag \& Co, Saarbrücken, Germany, 2009.

Negrete, A.: Constructing a comic to communicate scientific information about sustainable development and natural resources in Mexico, Social and Behavioral Sciences, 103, 200-209, 2013.

Negrete, A.: Tell me how much science you can tell: the RIRC method, Lambert Academic Publishing, Saarbrücken, Germany, 2014.

Negrete, A. and Lartigue, C.: The science of telling stories: Evaluating science communication via narratives (RIRC method), Journal of Media and Communication Studies, 2, 98-110, 2010.

Nelson, T. O. and Rothbart, R.: Acoustic savings for items forgotten from long-term memory, J. Exp. Psychol., 93, 357-360, 1972.

O'Brian, L.: Learn to remember, Duncan Baird Publishers, New York, USA, 2000.

Rios, P. and Negrete, A.: The object of art in science: Science communication via art installation, Journal of Science Communication, 12, 1-18, 2013.

Rupp, R.: Committed to memory, Aurum Press Ltd, New York, USA, 1998.

Shanklin, E.: Exploding lakes in myth and reality: an African case study, in: Myth and Geology, edited by: Piccardi, L. and Masse, W. B., Geological Society, London, Special Publications, 273, 165-176, 2007.

Squire, L. R.: Mechanisms of memory, Science, 232, 1612-1619, 1986.

Sternberg, R. J.: Cognitive psychology, Thomson Wadsworth, New York, USA, 2003.

Tulving, E.: Episodic and semantic memory, in: Organization of memory, edited by: Tulving E. and Donalson W., New York Academic Press, New York, USA, 1972.

Tulving, E. and Craik, F. I.: The Oxford handbook of memory, Oxford University Press, New York, USA, 2000.

Tulving, E. and Pearlstone, Z.: Availability versus accessibility of information in memory for words, J. Verb. Learn. Verb. Be., 5, 381-391, 1966.

Wells, H. G.: The time machine, Heinemann, London, UK, 1895.

Willis, C.: Science in science fiction: a writer's perspective, in: Science in science fiction, edited by: Stocker, J. H., 187-200, Longman, London, UK, 1998.

Wilton, R. N.: The structure of memory: evidence concerning the recall of surface and background colour shapes, Q. J. Exp. Psychol., 41A, 579-598, 1989.

Wilton, R. N.: The mediation of paired associate recall by representation of properties ascribed to objects in perception and imagination, Q. J. Exp. Psychol., 42A, 611-634, 1990.

Yates, F. A.: The art of memory, Pilmico Press, London, UK, 1992.

Zak, P. J., Stanton, A. A., and Ahmadi, S.: Oxytocin Increases Generosity in Humans, PLoS ONE, 2, e1128, https://doi.org/10.1371/journal.pone.0001128, 2007. 